Revista Matemática Universitária, vol. 1, 2019

ISSN: 2675-5254-DOI: https ://doi.org/10.21711/26755254/rmu202015

\title{
SOBRE A CLÁSSICA FUNÇÃO DE MITTAG-LEFFLER
}

\author{
EDMUNDO CAPELAS DE OLIVEIRA
}

\begin{abstract}
Resumo. Apresentamos e discutimos a clássica função de MittagLeffler como sendo uma possível generalização da função exponencial. Mencionamos algumas de suas possíveis generalizações, seja com o aumento do número de parâmetros, seja aumentando o número de variáveis ou ainda com o aumento de ambos, parâmetros e variáveis. Justificamos a sua importância junto ao cálculo de ordem não inteira e, como simples aplicações, efetuamos o cálculo de uma surpreendente soma e resolvemos uma equação diferencial fracionária.
\end{abstract}

\section{INTRODUÇÃO}

Em fins do Século XIX duas questões da Análise Matemática propiciaram estudos que mais tarde viriam a desempenhar papel central em vários campos da ciência: as integrais de Mellin-Barnes, introduzidas por Pincherle [48], em 1888, tendo sido suas propriedades estudadas por Mellin [34], enquanto Barnes [2] utilizou-as no estudo da função hipergeométrica; e a continuação analítica de funções holomorfas de uma variável complexa [35]. Neste trabalho, vamos nos concentrar na segunda questão, o procedimento da continuação analítica de séries de potência fora de seu disco de convergência, estudado por Mittag-Leffler (1846 - Gösta Magnus Mittag-Leffler - 1927) [35, 36, 37, 38, 39, 41] que propôs uma função, dependendo de um parâmetro complexo com parte real positiva, no integrando da chamada integral de Laplace-Abel [23].

Data de aceitação: 22 de setembro de 2019 (Data da publicação do artigo).

Palavras chave. Mittag-Leffler, Cálculo fracionário, Derivada de Caputo.

Agradeço ao revisor e ao editor pelas várias sugestões que deixaram o texto mais adequado. Ao revisor, também, por ter mencionado a referência [13] que possibilitou a inserção de várias outras referências importantes. 
Entre os anos de 1902 e 1905, Mittag-Leffler introduziu uma função [44], que hoje é conhecida como a clássica função de Mittag-Leffler, denotada por $E_{\alpha}(z)$ dependente de um parâmetro, através de uma série de potências contendo uma função gama como sendo uma generalização da função exponencial, e estudou várias de suas propriedades, em particular, aquelas associadas às séries divergentes, em uma série de artigos científicos $[40,42,43,44,45,46]$. É importante notar que neste período outras funções, relacionadas com a questão estudada por Mittag-Leffler, foram introduzidas, dentre elas mencionamos Le Roy [29], Lindelöf [31], Malmquist [32] e Wiman [64], cada uma com a sua particularidade [14]. Ainda mais, Hille \& Tamarkin [25], em 1930, como uma possível aplicação, apresentaram uma solução da equação tipo Abel-Volterra em termos da função de Mittag-Leffler.

Entre as décadas de 1940 e 1970 foram publicados, do ponto de vista estritamente matemático, vários trabalhos envolvendo séries de potências estudadas do ponto de vista das variáveis complexas, como funções inteiras, distribuição de zeros [3, 11, 12, 13, 19, 30, 51, 53, 59].

Ainda nos anos 1960, com a introdução da chamada função $H$ de Fox [18], ficou claro que a função de Mittag-Leffler pertencia a uma classe mais geral de funções que não se encaixavam nas clássicas funções especiais, em particular as funções hipergeométricas e hipergeométricas confluentes [5]. A função de Mittag-Leffler e suas correlatas, como as mencionadas anteriormente, bem como as funções hipergeométricas e hipergeométricas confluentes, são casos particulares da função $H$ de Fox [33]. É neste ponto que as integrais de Mellin-Barnes são importantes, pois a função $H$ de Fox é definida em termos de uma integral no plano complexo cujo integrando contém um quociente de funções gama.

O interesse pela função de Mittag-Leffler foi renovado e ganhou importância maior após a primeira conferência internacional sobre o cálculo fracionário, em 1974 [54], onde vários artigos ali apresentados, envolvendo a função de Mittag-Leffler, colocaram uma ordem no que hoje constitui-se um importante ramo da matemática, pois os estudos de temas no cálculo fracionário ganharam popularidade e confiança da comunidade científica, e continua em franca ascensão, tanto na teoria quanto nas inúmeras aplicações. Hoje, podemos dizer que a função de Mittag-Leffler adquiriu o status de uma função especial e já conta com livros e artigos dedicados exclusivamente ao seu estudo [22, 24, 52], bem como ganhou uma entrada no 2000 Mathematics Subjects Classification: 33E12-Mittag-Leffler functions and generalizations. Uma breve introdução à função de Mittag-Leffler, em língua portuguesa, pode ser encontrada em [17]. Enfim, aos interessados em métodos numéricos e/ou computacionais sugerimos [20, 21].

Assim como em todo campo de pesquisa, a generalização é sempre um fato. Na década de 1950, a partir dos trabalhos de Humbert [26], Agarwal [1] e Humbert \& Agarwal [27], uma nova função do tipo 
Mittag-Leffler foi mencionada, agora com dois parâmetros e que contém, como caso particular, a função de Mittag-Leffler e que, com os estudos de Wiman [64], hoje é conhecida como uma função de MittagLeffler com dois parâmetros, ainda que existam citações como função de Wiman. Na década de 1970, uma função de Mittag-Leffler contendo três parâmetros foi introduzida por Prabhakar [50] e que desempenha um papel importante em campos de pesquisa, em particular na solução de várias equações diferenciais fracionárias. E, claro, as generalizações não pararam por aí, porém nosso intuito é discutirmos apenas a função de Mittag-Leffler [7, 28, 55, 56].

Neste trabalho, na Seção 2, recuperamos vários resultados necessários que serão importantes para a continuação do trabalho; na Seção 3, apresentamos a função de Mittag-Leffler e as funções de Mittag-Leffler com dois e três parâmetros, enquanto na Seção 4, mostramos algumas propriedades das funções de Mittag-Leffler para, enfim, na Seção 5, discutir duas aplicações envolvendo uma particular soma associada à função de Mittag-Leffler com dois parâmetros bem como uma simples equação diferencial fracionária cuja solução é uma função de MittagLeffler. Concluímos apresentando as considerações finais.

\section{PRELIMINARES}

Ainda que este trabalho não pretenda ser um tratado sobre a função de Mittag-Leffler, duas importantes propriedades merecem destaque. A função de Mittag-Leffler é uma função inteira, analítica em todo o plano complexo, o que neste texto, por exemplo, justifica passagens ou demonstrações, e o conceito de função completamente monótona, propriedade essa que desempenha papel fundamental em várias aplicações [6]. Então, apresentamos as definições para uma função inteira $f(z)$ para, na próxima seção, particularizarmos para a função de Mittag-Leffler. Enfim, concluímos esta seção apresentando a definição da derivada fracionária no sentido de Caputo, bem como a expressão que fornece a transformada de Laplace dessa derivada [17], visando as aplicações.

2.1. Função inteira. Começamos por introduzir o conceito de função inteira. A fim de caracterizar uma função inteira, após o conceito de razão de crescimento, apresentamos os conceitos de ordem e tipo, duas medidas do crescimento no infinito.

DefiniçÃo 2.1. FunÇÃo Inteira. Seja $z \in \mathbb{C}$. Uma função $f: \mathbb{C} \rightarrow$ $\mathbb{C}$ é chamada inteira se ela é analítica $(\mathbb{C}$-diferenciável) em todo o plano complexo, i.e., em todo o ponto $z_{0} \in \mathbb{C}$ existe o limite

$$
\lim _{z \rightarrow z_{0}} \frac{f(z)-f\left(z_{0}\right)}{z-z_{0}} \in \mathbb{C}
$$


Ainda mais, é representada por uma série de potências convergente em todo $\mathbb{C}$

$$
f(z)=\sum_{k=0}^{\infty} a_{k} z^{k}
$$

sendo $a_{k}$ os coeficientes da série de potências.

Antes de apresentar a condição para garantir que essa série de potências converge, é importante mencionar o teorema de Liouville, bem como uma extensão incluindo um ponto no infinito, associado às funções transcendentais. O teorema de Liouville afirma que toda função inteira limitada é uma constante. Ainda mais, toda função que é inteira no plano complexo estendido (plano complexo e o ponto no infinito) é constante. No caso de uma função inteira não constante, essa apresenta uma singularidade no infinito, sendo uma singularidade polar (um polo), no caso de um polinômio, ou uma singularidade essencial, no caso de uma função inteira transcendente.

\section{TEOREMA 2.1. CritéRIO DE CONVERGÊNCIA.}

A condição necessária e suficiente para que a soma de uma série de potências represente uma função inteira é que os coeficientes $a_{k}$ satisfaçam a condição

$$
\lim _{k \rightarrow \infty}\left|a_{k}\right|^{\frac{1}{k}}=0
$$

isto é, o raio de convergência é infinito. Diante desse resultado, concluímos: toda série de potências satisfazendo essa condição representa uma função inteira.

Antes de apresentar os conceitos de ordem e tipo, visto serem características importantes a respeito do comportamento de uma função inteira, vamos definir razão de crescimento da função.

DefiniçÃo 2.2. RazÃo DE CRescimento. Sejam $z \in \mathbb{C}$ e $f(z)$ uma função complexa. Define-se a razão de crescimento por

$$
M_{f}(r)=\max _{|z|=r}|f(z)| \cdot
$$

Passemos, agora, a definir os conceitos de ordem, denotada por $\rho$, e tipo, denotado por $\sigma$, associados às funções inteiras, relativos ao crescimento no infinito. Por meio de um teorema, expressamos essas medidas em termos dos coeficientes da série de potências [30].

DEFINIÇÃO 2.3. ORDEM FINITA. Uma função inteira $f$ é dita de ordem finita, se existem números $c>0$ e $r>0$ tais que

$$
f(z) \leq \exp \left(|z|^{c}\right)
$$

para todo $|z|<r$. Se a função $f$ é de ordem $\rho$, seu crescimento no infinito é $\exp \left(|z|^{\rho}\right)$. 
DEFINIÇÃo 2.4. TiPo. O tipo de uma função inteira é, também, uma medida do crescimento no infinito para funções de mesma ordem. Se a função $f$ é de ordem $\rho$ e tipo $\sigma$ seu crescimento no infinito é $\exp \left(\sigma|z|^{\rho}\right)$.

TeOrema 2.2. ORDem E TIPO. A ordem e o tipo de uma função inteira são expressos em termos dos coeficientes da série de potências, $a_{n}$, através das expressões, respectivamente,

$$
\rho=-\varlimsup_{n \rightarrow \infty}\left(\frac{n \ln n}{\ln \left|a_{n}\right|}\right) \quad \text { e } \quad(\sigma e \rho)^{\frac{1}{\rho}}=\varlimsup_{n \rightarrow \infty}\left(n^{\frac{1}{\rho}}\left|a_{n}\right|^{\frac{1}{n}}\right)
$$

Podemos dizer, então, baseados nas DEFINIÇÃo 2.3 e DEFINIÇÃo 2.4 que o tipo é uma medida mais refinada, relativamente às funções de mesma ordem. Por exemplo, sejam $n \in \mathbb{N}, a>0$ e a função $f(z)=$ $\exp \left(a z^{n}\right)$ dizemos que essa função é de ordem $\rho=n$ e tipo $\sigma=a$.

2.2. Funções monótonas. São várias as aplicações em que a metodologia da transformada de Laplace desempenha papel fundamental. Por outro lado, as funções completamente monótonas são importantes, por exemplo, no estudo de modelos de viscoelasticidade. Existe uma conexão entre as transformadas de Laplace de funções completamente monótonas, isto é, as transformadas bilateriais de Laplace (Stieltjes) e as chamadas funções de Bernstein [60].

Começamos introduzindo o conceito de medida finita, relacionada com a transformada de Laplace, para, depois, introduzir o conceito de função completamente monótona.

DefiniÇÃo 2.5. TRANSFormada DE LAPlaCE. A transformada de Laplace de uma função $f:[0, \infty) \rightarrow[0, \infty)$ ou uma medida $\mu$ em $[0, \infty)$ é definida por

$$
\mathscr{L}(f, s)=\int_{0}^{\infty} e^{-s t} f(t) \mathrm{d} t \quad \text { ou } \quad \mathscr{L}(\mu, s)=\int_{[0, \infty)} e^{-s t} \mu(\mathrm{d} t)
$$

desde que as integrais convirjam.

ProposiçÃo 2.1. MEdidA FINITA. Seja $\mathscr{L}(\cdot)$ a transformada de Laplace. Uma medida $\mu$ em $[0, \infty)$ é finita se, e somente se, $\mathscr{L}\left(\mu, 0^{+}\right)<$ $\infty$. A medida $\mu$ é unicamente determinada por sua transformada de Laplace.

Medidas finitas são unicamente determinadas em termos de suas transformadas de Laplace. Diante disso, o conceito de função completamente monótona desempenha papel importante.

DEFiniÇÃo 2.6. FunÇÃo COMPletamente MONÓtona. Uma fun$c ̧ a \tilde{o} f:(0, \infty) \rightarrow \mathbb{R}$ é dita completamente monótona se possui derivadas $f^{(n)}(x)$ de todas as ordens, $n=0,1,2, \ldots$ e as derivadas alternando os sinais,

$$
(-1)^{n} f^{(n)}(x) \geq 0
$$

para todo $x \in(0, \infty)$. 
A equivalência da DEFINIÇÃo 2.6 com a existência de uma representação da função $f$ na forma de uma integral de Laplace-Stieltjes com densidade não decrescente e medida $\mathrm{d} \mu$ não negativa [63] é

$$
f(x)=\int_{0}^{\infty} e^{-x t} \mathrm{~d} \mu(t) .
$$

2.3. Derivada fracionária de Caputo. Existem várias maneiras de definir uma derivada fracionária, dentre elas aquela de Caputo. Essa derivada é dada como a integral fracionária de uma derivada de ordem inteira. Vamos definir essa derivada e apresentar a expressão que fornece a sua transformada de Laplace. Para outras formulações da derivada fracionária sugerimos o primeiro capítulo da referência [7] onde é apresentada uma linha do tempo relativa ao cálculo fracionário, e para a transformada de Laplace, ver [8, 10, 63].

Definição 2.7. Integral Fracionária. Sejam $\alpha>0$, a ordem $d a$ integral fracionária e $f(t)$ uma função (bem comportada) com $t \in \mathbb{R}^{+}$. Definimos a integral fracionária, também conhecida como integral de Riemann-Liouville, de ordem $\alpha$, pela expressão

$$
J^{\alpha} f(t):=\frac{1}{\Gamma(\alpha)} \int_{0}^{t}(t-\tau)^{\alpha-1} f(\tau) \mathrm{d} \tau
$$

com $t>0$ e $\alpha>0$. Ainda mais, definimos $J^{0}:=\mathbb{I}$ sendo $\mathbb{I}$ o operador identidade, $J^{0} f(t)=f(t)$.

DefiniÇÃo 2.8. Derivada Fracionária de CAputo. Consideremos $m-1<\alpha \leq m$ com $m=1,2,3, \ldots$ Definimos o operador derivada fracionária de Caputo, denotado por ${ }^{C} \mathrm{D}^{\alpha}$, a partir da expressão

$$
{ }^{C} \mathrm{D}^{\alpha}:=J^{m-\alpha} \mathrm{D}^{m}
$$

onde $J^{\alpha}$ é o operador integral fracionário e $\mathrm{D}^{m}$ o operador derivada de ordem inteira.

Utilizando a DEFINIÇÃO 2.7, podemos escrever

$$
{ }^{C} \mathrm{D}^{\alpha} f(t):= \begin{cases}\frac{1}{\Gamma(m-\alpha)} \int_{0}^{t} \frac{\mathrm{D}^{m} f(\tau)}{(t-\tau)^{\alpha+1-m}} \mathrm{~d} \tau, & m-1<\alpha<m, \\ \frac{\mathrm{d}^{\mathrm{m}}}{\mathrm{d} t^{m}} f(t), & \alpha=m .\end{cases}
$$

É importante notar que a derivada fracionária de Caputo de ordem $\alpha$ requer a integrabilidade absoluta da derivada de ordem inteira, $m$.

Propriedade 2.1. Transformada DE LAPlaCE DA DERIVAdA DE CAPuto. Consideremos uma função $f(t)$, bem comportada, com $t \in$ $\mathbb{R}^{+}$e $s=\sigma+i \tau$ com $\sigma, \tau \in \mathbb{R}$, o parâmetro da transformada. Admitamos que $f(t) e^{-s t} \rightarrow 0$ e todas as derivadas $f^{(i)} e^{-s t} \rightarrow 0 \mathrm{com}$ 
E. Capelas de Oliveira

$i=1,2,3, \ldots$ quando $t \rightarrow \infty$. A transformada de Laplace da derivada fracionária de Caputo é dada por

$$
\mathscr{L}\left[{ }^{C} \mathrm{D}^{\alpha} f(t)\right]=s^{\alpha} \mathscr{L}[f(t)]-\sum_{k=0}^{m-1} f^{(k)}\left(0^{+}\right) s^{\alpha-1-k}
$$

sendo $\alpha>0$ a ordem com $m-1<\alpha \leq m$.

Note que, se $\alpha=m=1,2, \ldots$ obtemos exatamente a expressão que fornece a transformada de Laplace da derivada de ordem inteira, $m$.

\section{A Clássica funçÃo de Mittag-Leffler}

A clássica função de Mittag-Leffler, a partir de agora, apenas função de Mittag-Leffler, contendo um parâmetro pode ser considerada como a mais simples generalização não trivial da função exponencial. Assim como a função exponencial é solução de uma equação diferencial ordinária com coeficientes constantes, a função de Mittag-Leffler é solução de uma equação diferencial fracionária. Devido a sua importância e abrangência na solução de várias equações diferenciais fracionárias, Mainardi cunhou-a de a rainha das funções especiais do cálculo fracionário [22]. Então, podemos afirmar que a função de Mittag-Leffler está para o cálculo fracionário assim como a função exponencial está para o cálculo de ordem inteira [17].

Definição 3.1. FunÇÃo De Mittag-Leffrler. Sejam $z \in \mathbb{C} e \alpha$ um parâmetro complexo com $\operatorname{Re}(\alpha)>0$. A função de Mittag-Leffler é definida a partir da série de potências

$$
E_{\alpha}(z)=\sum_{k=0}^{\infty} \frac{z^{k}}{\Gamma(\alpha k+1)}
$$

onde $\Gamma(\cdot)$ é uma função gama, também chamada de função de Euler de segunda espécie [5].

Vale a pena mencionar que vários autores optam por definir a função de Mittag-Leffler considerando a mudança $z \rightarrow z^{\alpha}$ e com as mesmas restrições impostas na DEFINIÇÃO 3.1, pois em ambos os casos, para $\alpha=1$, recuperamos a função exponencial.

A fim de discutir a convergência da série, podemos utilizar o teste da razão e um resultado envolvendo o quociente de duas funções gama [18] e que nos leva a concluir que a série converge em todo o plano complexo com a condição $\operatorname{Re}(\alpha)>0$. Para os demais casos $\operatorname{Re}(\alpha)<0$ (a série diverge) e $\operatorname{Re}(\alpha)=0$ e $|z|<1$ obtemos a soma geométrica. Daqui para a frente, vamos nos concentrar apenas no caso em que $\operatorname{Re}(\alpha)>0$, pois a função de Mittag-Leffler é uma função inteira, admite derivadas de todas as ordens. Ainda mais, quando o parâmetro é $\alpha=1$, vale a 
relação $\Gamma(k+1)=k$ ! de onde segue

$$
E_{1}(z)=\sum_{k=0}^{\infty} \frac{z^{k}}{k !}
$$

que é exatamente a função exponencial $E_{1}(z)=e^{z}$ e, portanto, é natural interpretar a função de Mittag-Leffler como uma possível generalização da função exponencial. Ainda mais, considerando o parâmetro $\alpha=2$ na Eq.(1), obtemos uma representação em série para o cosseno hiperbólico,

$$
E_{2}\left(z^{2}\right)=\sum_{k=0}^{\infty} \frac{z^{2 k}}{\Gamma(2 k+1)}=\sum_{k=0}^{\infty} \frac{z^{2 k}}{(2 k) !}=\cosh z .
$$

Diante dessas duas últimas expressões, no intervalo $1 \leq \alpha \leq 2$, a função de Mittag-Leffler interpola as funções exponencial e cosseno hiperbólico. Com a substituição $z^{2} \rightarrow-z^{2}$, obtemos uma expressão similar para a função cosseno trigonométrico. Por outro lado, não podemos expressar a função seno hiperbólico (trigonométrico) em termos de uma função de Mittag-Leffler o que só se torna possível com a introdução da função de Mittag-Leffler com dois parâmetros.

Antes de passarmos à função de Mittag-Leffler com dois parâmetros, vamos mostrar que a função de Mittag-Leffler é uma função inteira de ordem $\rho=\frac{1}{\alpha}$ e tipo $\sigma=1$, bem como mencionar que essa função é completamente monótona no eixo real negativo.

Proposição 3.1. FunçÃo De MitTag-Leffler. A função de MittagLeffler, como apresentada na DEFINIÇÃO 3.1, é uma função inteira, de ordem $\rho=\frac{1}{\alpha}$ e tipo $\sigma=1$.

Prova 3.1. A fim de mostrar que a função de Mittag-Leffler é uma função inteira, primeiramente identificamos os coeficientes da expansão em série de potências $a_{n}=1 / \Gamma(\alpha n+1) \operatorname{com} \alpha>0$. Utilizando o TEOREMA 2.1, devemos calcular o limite

$$
\lim _{n \rightarrow \infty}\left|\frac{1}{\Gamma(\alpha n+1)}\right|^{\frac{1}{n}} .
$$

Primeiramente, a partir da fórmula assintótica de Stirling,

$$
\Gamma(\alpha n+1)=\sqrt{2 \pi}(\alpha n)^{\alpha n+\frac{1}{2}} e^{-\alpha n}[1+O(1)], \quad n \rightarrow \infty
$$

podemos escrever para o limite

$$
\lim _{n \rightarrow \infty}\left|\frac{1}{\sqrt{2 \pi}(\alpha n)^{\alpha n+\frac{1}{2}} e^{-\alpha n}}\right|^{\frac{1}{n}}=\lim _{n \rightarrow \infty}\left|\frac{e^{\alpha}}{\sqrt{2 \pi}(\alpha n)^{\alpha+\frac{1}{2 n}}}\right| \rightarrow 0 .
$$

Então, visto que o limite é zero, a função é inteira. Agora, para mostrarmos que a ordem da função de Mittag-Leffler é dada por $\rho=\frac{1}{\alpha}$, 
usamos o TEOREMA 2.2, a fim de calcular o limite

$$
\rho=\varlimsup_{n \rightarrow \infty}\left(\frac{n \ln n}{\ln |\Gamma(\alpha n+1)|}\right) .
$$

Vamos utilizar, para $\alpha>0$, mais uma vez a fórmula assintótica de Stirling de onde segue o limite

$$
\rho=\lim _{n \rightarrow \infty}\left[\frac{n \ln n}{\ln \sqrt{2 \pi \alpha n}+\alpha n(\ln \alpha n-\ln e)}\right]
$$

que, utilizando a regra de l'Hôpital, fornece

$$
\rho=\frac{1}{\alpha}
$$

que é o resultado desejado, isto é, a função de Mittag-Leffler é uma função inteira e de ordem $\rho=\frac{1}{\alpha}$. Para mostrar que a função de MittagLeffler é do tipo $\sigma=1$ novamente utilizamos o TEOREMA 2.2, logo devemos calcular o limite, já com $\rho=\frac{1}{\alpha}$,

$$
\left(\sigma e \frac{1}{\alpha}\right)^{\alpha}=\varlimsup_{n \rightarrow \infty}\left(n^{\alpha} \frac{1}{[\Gamma(\alpha n+1)]^{\frac{1}{n}}}\right) .
$$

Utilizando a fórmula assintótica de Stirling e rearranjando, podemos escrever

$$
\sigma^{\alpha}=\lim _{n \rightarrow \infty}\left(\frac{1}{n}\right)^{\frac{1}{2 n}}=\lim _{x \rightarrow 0}\left(x^{x}\right)^{\frac{1}{2}} .
$$

Para calcular esse limite, utilizamos propriedades do logaritmo, de onde segue $\sigma=1$, isto é, a função de Mittag-Leffler é uma função inteira, de ordem $\rho=\frac{1}{\alpha}$ e tipo $\sigma=1$.

Proposição 3.2. Completamente monótona. A função de MittagLeffler, $E_{\alpha}(-x)$, conforme DEFINIÇÃO 3.1, com o parâmetro $\alpha$, tal que $0<\alpha \leq 1$ é completamente monótona para $x>0$.

Prova 3.2. Ver Pollard [49].

DefiniÇÃo 3.2. FunÇÃo DE MitTaG-LefFler COM DOIS PARÂMeTROs. Sejam $z \in \mathbb{C}$ e $\alpha$ e $\beta$ dois parâmetros complexos com $\operatorname{Re}(\alpha)>0$. A função de Mittag-Leffler com dois parâmetros é definida a partir da série de potências

$$
E_{\alpha, \beta}(z)=\sum_{k=0}^{\infty} \frac{z^{k}}{\Gamma(\alpha k+\beta)}
$$

onde $\Gamma(\cdot)$ é uma função gama.

Em analogia ao anterior, a função de Mittag-Leffler com dois parâmetros generaliza a função de Mittag-Leffler, pois para o parâmetro 
$\beta=1$ podemos escrever

$$
E_{\alpha, 1}(z)=\sum_{k=0}^{\infty} \frac{z^{k}}{\Gamma(\alpha k+1)}=E_{\alpha}(z)
$$

que é exatamente a função de Mittag-Leffler.

Como já mencionado, podemos expressar o seno hiperbólico (trigonométrico, com a mudança $z^{2} \rightarrow-z^{2}$ ) em termos da função de MittagLeffler com dois parâmetros,

$$
z E_{2,2}\left(z^{2}\right)=z \sum_{k=0}^{\infty} \frac{z^{k}}{\Gamma(2 k+2)}=\sum_{k=0}^{\infty} \frac{z^{2 k+1}}{(2 k+1) !}=\operatorname{senh} z \cdot
$$

Como a função de Mittag-Leffler é um caso particular da função de Mittag-Leffler com dois parâmetros, podemos afirmar que tanto o cosseno quanto o seno hiperbólicos (trigonométricos, com a mudança $z^{2} \rightarrow$ $\left.-z^{2}\right)$ podem ser expressos em termos de uma função de Mittag-Leffler com dois parâmetros. Apenas para mencionar, também podemos definir os chamados seno e cosseno fracionários, em termos de funções de Mittag-Leffler com dois parâmetros [17].

Definição 3.3. FunÇÃO DE MitTag-LefFler COM TRÊS PARÂMetros. Sejam $z \in \mathbb{C} e \alpha, \beta$ e $\gamma$ três parâmetros complexos com $\operatorname{Re}(\alpha)>0$. A função de Mittag-Leffler com três parâmetros é definida a partir da série de potências

$$
E_{\alpha, \beta}^{\gamma}(z)=\sum_{k=0}^{\infty} \frac{(\gamma)_{k}}{\Gamma(\alpha k+\beta)} \frac{z^{k}}{k !}
$$

onde $\Gamma(\cdot)$ é uma função gama e $(\gamma)_{k}$ o símbolo de Pochhammer [17].

Em analogia ao anterior, a função de Mittag-Leffler com três parâmetros generaliza a função de Mittag-Leffler com dois parâmetros, pois para o parâmetro $\gamma=1$ podemos escrever

$$
E_{\alpha, \beta}^{1}(z)=\sum_{k=0}^{\infty} \frac{(1)_{k}}{\Gamma(\alpha k+\beta)} \frac{z^{k}}{k !}=\sum_{k=0}^{\infty} \frac{z^{k}}{\Gamma(\alpha k+\beta)}=E_{\alpha, \beta}(z)
$$

que é exatamente a função de Mittag-Leffler com dois parâmetros.

A fim de concluir a seção vamos enunciar uma propriedade formal entre a função de Mittag-Leffler com três parâmetros com a derivada de ordem $n, n \in \mathbb{N}$, da função de Mittag-Leffler com dois parâmetros e, com isso, justificar a preferência de se trabalhar com a função de Mittag-Leffler com três parâmetros, em vez de com a derivada da função de Mittag-Leffler com dois parâmetros [17].

ProposiçÃo 3.3. RelaÇÃo entre funÇÕes De Mittag-LefFler. Sejam $n=0,1,2, \ldots, \alpha, \beta$ e $\gamma$ três parâmetros complexos com $\operatorname{Re}(\alpha)>$ 0. Consideremos $E_{\alpha, \beta}(\cdot)$ e $E_{\alpha, \beta}^{\gamma}(\cdot)$, respectivamente, as funçôes de 
E. Capelas de Oliveira

Mittag-Leffler com dois e três parâmetros, e a notação para a derivada de ordem $n$ dada por $E_{\alpha, \beta}^{(n)}(z)=\frac{\partial^{n}}{\partial z^{n}} E_{\alpha, \beta}(z)$. Vale a relação

$$
E_{\alpha, \beta}^{(n)}(z)=n ! E_{\alpha, \beta+\alpha k}^{k+1}(z) .
$$

Prova 3.3. Ver [17].

Enfim, vale a pena enfatizar que, como já mencionado, existem outras possibilidades para generalizações, seja quanto ao número de parâmetros, como fizemos com dois e três parâmetros, seja quanto ao aumento do número de variáveis [7, 28, 55, 56].

\section{Propriedades das funções de Mittag-Leffler}

Nesta seção, vamos nos concentrar no estudo das mais simples propriedades da função de Mittag-Leffler, ainda que existam muitas outras [17, 22]. Em particular, mostramos uma relação com a derivada de ordem $n$, com $n=0,1,2, \ldots$ e uma relação de recorrência envolvendo as funções de Mittag-Leffler clássica e com dois parâmetros.

Propriedade 4.1. Derivada De Ordem $n$. Sejam $z \in \mathbb{C}$ e $n=$ $0,1,2, \ldots$ Vale a relação

$$
\left(\frac{\mathrm{d}}{\mathrm{d} z}\right)^{n} E_{n}\left(z^{n}\right)=E_{n}\left(z^{n}\right)
$$

Prova 4.1. Vamos, partindo do primeiro membro, calcular a derivada de ordem $n$ da função de Mittag-Leffler, escrita em termos de uma série de potências,

$$
\left(\frac{\mathrm{d}}{\mathrm{d} z}\right)^{n} E_{n}\left(z^{n}\right)=\left(\frac{\mathrm{d}}{\mathrm{d} z}\right)^{n} \sum_{k=0}^{\infty} \frac{z^{n k}}{\Gamma(n k+1)} .
$$

Visto que a série é convergente, podemos comutar a derivada com a série, $\log o$

$$
\left(\frac{\mathrm{d}}{\mathrm{d} z}\right)^{n} E_{n}\left(z^{n}\right)=\sum_{k=0}^{\infty} \frac{1}{\Gamma(n k+1)}\left(\frac{\mathrm{d}}{\mathrm{d} z}\right)^{n} z^{n k} .
$$

Utilizando a expressão para a derivada de ordem $n$ de uma potência de ordem nk, obtemos

$$
\left(\frac{\mathrm{d}}{\mathrm{d} z}\right)^{n} E_{n}\left(z^{n}\right)=\sum_{k=1}^{\infty} \frac{1}{\Gamma(n k+1)} \frac{(n k) !}{(n k-n) !} z^{n k-n}
$$

onde alteramos o indice de soma, pois $1 / \Gamma(-k) \rightarrow 0$. A partir da relação entre o fatorial e a função gama, a expressão anterior permite escrever

$$
\left(\frac{\mathrm{d}}{\mathrm{d} z}\right)^{n} E_{n}\left(z^{n}\right)=\sum_{k=1}^{\infty} \frac{1}{\Gamma(n k+1)} \frac{\Gamma(n k+1)}{\Gamma(n k-n+1)} z^{n k-n} .
$$


Simplificando a expressão anterior e mudando o indice de soma $k \rightarrow$ $k+1$ obtemos

$$
\left(\frac{\mathrm{d}}{\mathrm{d} z}\right)^{n} E_{n}\left(z^{n}\right)=\sum_{k=0}^{\infty} \frac{1}{\Gamma(n k+1)} z^{n k}=E_{n}\left(z^{n}\right)
$$

que é exatamente o resultado desejado.

Propriedade 4.2. Fórmula de DuplicaÇão. Sejam $z \in \mathbb{C}$ e $\alpha$ um parâmetro complexo com $\operatorname{Re}(\alpha)>0$. A fórmula de duplicação é dada pela expressão

$$
E_{\alpha}(z)+E_{\alpha}(-z)=2 E_{2 \alpha}\left(z^{2}\right)
$$

PROVA 4.2. Vamos expressar o primeiro membro em termos das respectivas séries de potências

$$
E_{\alpha}(z)+E_{\alpha}(-z)=\sum_{k=0}^{\infty} \frac{z^{k}}{\Gamma(\alpha k+1)}+\sum_{k=0}^{\infty} \frac{(-z)^{k}}{\Gamma(\alpha k+1)}
$$

que, rearranjando num único somatório, permite escrever

$$
E_{\alpha}(z)+E_{\alpha}(-z)=\sum_{k=0}^{\infty} \frac{z^{k}+(-z)^{k}}{\Gamma(\alpha k+1)} .
$$

Visto que para $k$ um número impar a soma é sempre zero, podemos mudar o indice de soma somente para os números pares, logo introduzimos $k \rightarrow 2 k$, de onde podemos escrever

$$
E_{\alpha}(z)+E_{\alpha}(-z)=2 \sum_{k=0}^{\infty} \frac{z^{2 k}}{\Gamma(2 \alpha k+1)}
$$

que, voltando com a função de Mittag-Leffler, fornece

$$
E_{\alpha}(z)+E_{\alpha}(-z)=2 E_{2 \alpha}\left(z^{2}\right)
$$

que é exatamente o resultado desejado.

Propriedade 4.3. RelaÇÃo De ReCorrênCIA. Sejam $z \in \mathbb{C} e$ $\alpha$ e $\beta$ dois parâmetros complexos com $\operatorname{Re}(\alpha)>0$. Vale a relação de recorrência envolvendo a derivada primeira da função de Mittag-Leffler com dois parâmetros

$$
\frac{\mathrm{d}}{\mathrm{d} z} E_{\alpha, \beta}(z)=\left(z \frac{\mathrm{d}}{\mathrm{d} z}+1\right) E_{\alpha, \alpha+\beta}(z) \cdot
$$

Prova 4.3. Começamos com a definição da função de Mittag-Leffler com dois parâmetros escrita em termos de uma série de potências,

$$
E_{\alpha, \beta}(z)=\sum_{k=0}^{\infty} \frac{z^{k}}{\Gamma(\alpha k+\beta)}
$$


cuja derivada primeira permite escrever para o primeiro membro, já alterando o indice de soma

$$
\mathrm{I}_{M}=\frac{\mathrm{d}}{\mathrm{d} z} \sum_{k=0}^{\infty} \frac{z^{k}}{\Gamma(\alpha k+\beta)}=\sum_{k=0}^{\infty} \frac{(k+1) z^{k}}{\Gamma(\alpha k+\alpha+\beta)} .
$$

Separando em duas somas e escrevendo a segunda delas em termos da função de Mittag-Leffler com dois parâmetros, obtemos

$$
\mathrm{I}_{M}=\sum_{k=0}^{\infty} \frac{k z^{k}}{\Gamma(\alpha k+\alpha+\beta)}+E_{\alpha, \alpha+\beta}(z) .
$$

Podemos escrever para a soma remanescente, em termos da derivada, a expressão

$$
\mathrm{I}_{M}=z \frac{\mathrm{d}}{\mathrm{d} z} \sum_{k=0}^{\infty} \frac{z^{k}}{\Gamma(\alpha k+\alpha+\beta)}+E_{\alpha, \alpha+\beta}(z)
$$

de onde segue, já escrevendo em termos da função de Mittag-Leffler com dois parâmetros, a seguinte relação

$$
\mathrm{I}_{M}=z \frac{\mathrm{d}}{\mathrm{d} z} E_{\alpha, \alpha+\beta}(z)+E_{\alpha, \alpha+\beta}(z)
$$

ou ainda, na conveniente forma

$$
\mathrm{I}_{M}=\left(z \frac{\mathrm{d}}{\mathrm{d} z}+1\right) E_{\alpha, \alpha+\beta}(z)
$$

que é exatamente o segundo membro.

Propriedade 4.4. RELAÇÃo COM A FUnÇÃo HIPERGEOMÉtRICA CONFluente. Sejam $z \in \mathbb{C}$ e $\beta$ um parâmetro complexo com $\beta \neq$ $0,-1,-2, \ldots$ A relaçãa entre uma particular função de Mittag-Leffler, $E_{1, \beta}(z)$, e a função hipergeométrica confluente, denotada por ${ }_{1} F_{1}(a ; c ; z)$, com a e c parâmetros complexos, é dada pela relação [17]

$$
\Gamma(\beta) E_{1, \beta}(z)={ }_{1} F_{1}(1 ; \beta ; z) \text {. }
$$

Prova 4.4. Introduzindo o parâmetro $\alpha=1$ na expressão em série para a função de Mittag-Leffler com dois parâmetros, podemos escrever para o primeiro membro

$$
\mathrm{I}_{M}=\Gamma(\beta) E_{1, \beta}(z)=\Gamma(\beta) \sum_{k=0}^{\infty} \frac{z^{k}}{\Gamma(k+\beta)} .
$$

Da definição do simbolo de Pochhammer, o segundo membro da precedente pode ser escrito na forma

$$
\mathrm{I}_{M}=\Gamma(\beta) E_{1, \beta}(z)=\Gamma(\beta) \sum_{k=0}^{\infty} \frac{(1)_{k}}{(\beta)_{k}} \frac{z^{k}}{k !}
$$


que, identificando com a expressão em série para a função hipergeométrica confluente

$$
{ }_{1} F_{1}(a ; c ; z)=\sum_{k=0}^{\infty} \frac{(a)_{k}}{(c)_{k}} \frac{z^{k}}{k !}
$$

fornece a seguinte relação

$$
\mathrm{I}_{M}={ }_{1} F_{1}(1 ; \beta ; z)
$$

de onde segue a relação entre a função de Mittag-Leffler com dois parâmetros e a função hipergeométrica confluente,

$$
\Gamma(\beta) E_{1, \beta}(z)={ }_{1} F_{1}(1 ; \beta ; z)
$$

que é o resultado desejado.

Existem muitas outras relações, além dessas, em particular as representações integrais e o estudo das funções nos extremos, as chamadas expressões assintóticas, distribuição de zeros e relações com outras funções especiais, por exemplo, mostrar que a função de Mittag-Leffler é um caso particular de uma função $H$ de Fox, porém isso foge do escopo desta introdução [22].

Passemos a estudar duas aplicações envolvendo as funções de MittagLeffler admitindo, a partir de agora, $z=x \in \mathbb{R}$, isto é, a função é tal que o seu argumento é uma variável real.

\section{APLiCAÇÕES}

Como aplicações das funções de Mittag-Leffler, vamos discutir o cálculo de uma surpreendente soma envolvendo a função de Mittag-Leffler com dois parâmetros [9] e uma equação diferencial fracionária com coeficientes constantes, ambas fazendo uso da metodologia da transformada de Laplace, isto é, transformamos o problema de partida, equação diferencial e condições iniciais e, a fim de recuperarmos a sua solução, utilizamos a respectiva transformada de Laplace inversa.

APliCAÇÃo 5.1. Soma DE FunÇÕES DE MitTaG-LefFler COM DOIS PARÂmetros. Sejam $x \in \mathbb{R}$ e $n \in \mathbb{N}$. Mostre que a soma

$$
\sum_{k=1}^{n} x^{k-1} E_{n, k}\left(x^{n}\right)
$$

é independente de $n$.

RESOLUÇÃO 5.1. Introduzimos a notação $\mathrm{I}_{\mathrm{M}}=\sum_{k=1}^{n} x^{k-1} E_{n, k}\left(x^{n}\right)$. Tomando a transformada de Laplace de ambos os membros, temos

$$
\mathscr{L}\left[\mathrm{I}_{\mathrm{M}}\right]=\mathscr{L}\left\{\sum_{k=1}^{n} x^{k-1} E_{n, k}\left(x^{n}\right)\right\} .
$$


Utilizando a representação em série para a função de Mittag-Leffler e rearranjando obtemos

$$
\mathscr{L}\left[\mathrm{I}_{\mathrm{M}}\right]=\sum_{k=1}^{n} \sum_{\ell=0}^{\infty} \frac{1}{\Gamma(n \ell+k)} \int_{0}^{\infty} x^{n \ell+k-1} e^{-s x} \mathrm{~d} x .
$$

Efetuando a mudança de variável $s x=t$, considerando a definição da função gama e simplificando podemos escrever

$$
\mathscr{L}\left[\mathrm{I}_{\mathrm{M}}\right]=\sum_{k=1}^{n} \frac{1}{s^{k}} \sum_{\ell=0}^{\infty}\left(\frac{1}{s^{n}}\right)^{\ell} .
$$

O segundo somatório, em $\ell$, é a soma dos infinitos termos de uma progressão geométrica de primeiro termo unitário e razão $s^{-n}$, enquanto o somatório em $k$ é a soma dos $n$ primeiros termos de uma progressão geométrica de primeiro termo e razão iguais a $\mathrm{s}^{-1}$, logo

$$
\mathscr{L}\left[\mathrm{I}_{\mathrm{M}}\right]=\frac{1}{s-1} .
$$

Tomando a transformada de Laplace inversa de ambos os membros podemos escrever

$$
\mathrm{I}_{\mathrm{M}}=\mathscr{L}\left[\frac{1}{s-1}\right]=e^{x}
$$

ou ainda, da seguinte forma

$$
\sum_{k=1}^{n} x^{k-1} E_{n, k}\left(x^{n}\right)=e^{x}
$$

que é o resultado desejado.

Enfim, admitamos que a equação diferencial fracionária é uma extensão natural da equação diferencial ordinária, tendo em mente a mudança que fizemos quando introduzimos a função de Mittag-Leffler, apresentando-a como uma generalização da função exponencial, ou seja, no lugar do fatorial na série de potências, temos uma função gama. Com isso queremos dizer que, formalmente, basta substituir no lugar da ordem inteira da derivada na equação diferencial uma ordem arbitrária e, ao final, o resultado conhecido deve ser recuperado.

APLICAÇÃO 5.2. EQUAÇÃO DIFERENCIAL DE ORDEM $\mu$ COM $1<\mu \leq$ 2. Sejam $y(x)$ uma função continua, $y(0)=A$ e $y^{\prime}(0)=B$ duas constantes positivas e $\mu$ um parâmetro tal que $1<\mu \leq 2$. Resolver a equação diferencial fracionária de ordem $\mu$

$$
\frac{\mathrm{d}^{\mu}}{\mathrm{d} x^{\mu}} y(x)+k^{2} y(x)=0
$$

sendo $k^{2}>0$ uma constante e a derivada sendo considerada no sentido de Caputo, conforme DEFINIÇÃO 2.8. 
Ainda que não tenhamos mencionado, esta equação diferencial ordinária no caso em que $\mu=2$ é a equação diferencial associada ao movimento harmônico simples, com solução dada em termos de funções trigonométricas, senos e cossenos, e dependendo das condições iniciais, podemos obter uma particular solução satisfazendo o problema de valor inicial. Ora, parece natural a extensão, considerando a ordem $\mu$, não inteira, a fim de que tenhamos uma equação diferencial fracionária. Note que excluímos o outro extremo, pois apenas um dos extremos deve ser considerado para recuperar o caso inteiro. Claro, poderíamos ter considerado apenas o outro extremo, excluindo o lado direito do intervalo, porém não teríamos, no caso limite, uma equação diferencial de ordem dois, representando um movimento ondulatório. Ao final da resolução vamos mencionar o caso $\mu=1$.

RESOLUÇÃO 5.2. A fim de resolver essa equação diferencial fracionária, vamos utilizar a metodologia da transformada de Laplace. Então, tomando a transformada de Laplace de ambos os membros da equação diferencial fracionária temos

$$
\mathscr{L}\left[\frac{\mathrm{d}^{\mu}}{\mathrm{d} x^{\mu}} y(x)\right]+k^{2} \mathscr{L}[y(x)]=0 .
$$

Utilizando a PROPRIEDADE 2.1, com $m=2$, obtemos a seguinte equação algébrica, para $F(s)$,

$$
\underbrace{s^{\mu} F(s)-y(0) s^{\mu-1}-y^{\prime}(0) s^{\mu-2}}_{*}+k^{2} F(s)=0
$$

onde $F(s)$ é a transformada de Laplace de $y(x)$ com parâmetro s. Note que, como já havíamos mencionado, no caso em que $\mu=2$, a expressão destacada com * nada mais é que a expressão para a transformada de Laplace da derivada de ordem dois.

Substituindo as condiçôes iniciais, $y(0)=A$, relativa a um deslocamento inicial, $y^{\prime}(0)=B$, associada a uma velocidade inicial, e resolvendo a equação algébrica, temos sua solução dada por

$$
F(s)=A \frac{s^{\mu-1}}{s^{\mu}+k}+B \frac{s^{\mu-2}}{s^{\mu}+k} .
$$

A fim de proceder com a inversão da transformada, devemos utilizar o plano complexo [4, 7] de onde segue para a solução do problema, composto pela equação diferencial e as condições iniciais

$$
y(x)=A E_{\mu}\left(k x^{\mu}\right)+B E_{\mu, 2}\left(-k x^{\mu}\right)
$$

com $E_{\mu}(\cdot)$ a função de Mittag-Leffler e $E_{\mu, 2}(\cdot)$ a função de MittagLeffler com dois parâmetros.

Como já mencionamos, vamos fazer um comentário relativo a uma possível aplicação num problema real. No caso em que $\mu=2$, recuperamos o resultado do oscilador harmônico livre e, por extensão de 
E. Capelas de Oliveira

linguagem, dizemos que a solução obtida, conforme Eq.(4), é a solução da equação diferencial fracionária descrevendo o movimento do oscilador harmônico fracionário.

No caso em que acrescentamos um termo de derivada de ordem um, resulta na equação associada ao movimento do oscilador amortecido e podemos obter uma relação entre o coeficiente de amortecimento e o parâmetro que indica a ordem da equação diferencial fracionária [7].

Ainda que não esteja no intervalo, no particular caso em que $\mu \rightarrow 1$, ordem inteira, a equação torna-se de ordem um, e podemos explicitar a solução em termos de funções exponencial e trigonométrica.

Se, por outro lado, tivéssemos considerado o intervalo $0<\mu \leq 1$, poderíamos associar a um problema envolvendo o chamado decaimento radioativo fracionário, visto que o caso inteiro do parâmetro $\mu=1$, descreve o movimento de um processo de decaimento.

Concluímos mencionando um estudo da equação diferencial fracionária associado ao clássico problema do telégrafo onde a solução é dada em termos de uma função de Mittag-Leffler com três parâmetros [15], bem como o clássico estudo envolvendo a função de Green fracionária [16].

\section{Conclusões}

Apresentamos um simples estudo motivando o uso da função de Mittag-Leffler, visando abordar problemas advindos do cálculo fracionário. Após a introdução da função de Mittag-Leffler, hoje completamente consolidada como uma função especial, apresentamos algumas propriedades e estendemos essa função para o caso com dois e três parâmetros, a fim de obter as funções de Mittag-Leffler com dois e com três parâmetros, respectivamente, além de mencionar outras possibilidades para mais extensões, seja quanto ao número de parâmetros, seja quanto ao número de variáveis ou ambos, tanto o número de parâmetros quanto o número de variáveis.

A partir da metodologia da transformada de Laplace, como aplicações, discutimos o cálculo de uma série envolvendo uma função de Mittag-Leffler e a resolução de um problema de valor inicial, contendo uma equação diferencial ordinária fracionária e duas condições iniciais, recuperando, como caso particular, o caso do oscilador harmônico livre.

Hoje, como já mencionado, o cálculo fracionário está completamente consolidado e existem várias frentes para serem abordadas, dentre elas mencionamos aquelas no campo estritamente teórico, onde a chamada derivada fracionária $\psi$-Hilfer [61, 62] desempenha papel importante o estudo da chamada derivada de Hilfer-Katugampola [47] onde a clássica derivada de Caputo-Hadamard é um caso particular e o estudo da monotonicidade da chamada função de Prabhakar, dada como o produto de uma particular potência do argumento de uma função de Mittag-Leffler por uma função de Mittag-Leffler com três parâmetros, 
por exemplo, associada ao estudo da relaxação anômala [6], dentre outras.

Enfim, para aqueles interessados nas várias formas de se apresentar um operador associado a uma derivada fracionária sugerimos $[57,58]$ e as referências ali contidas.

\section{REFERÊNCIAS}

[1] Ravi P. Agarwal, A propos d'une note de M. Pierre Humbert, C. R. Acad. Sci. Paris 236, 2031-2032 (1953).

[2] Ernest William Barnes, A new development in the theory of the hypergeometric functions, Proc. London Math. Soc., 6, 141-177, (1908).

[3] Ralph Philip Boas Jr., Entire Functions, Academic Press. Inc., Publishers, NY (1954).

[4] Edmundo Capelas de Oliveira e Waldyr Alves Rodrigues Jr., Funções Analíticas com Aplicações, Editora Livraria da Física, São Paulo (2005).

[5] Edmundo Capelas de Oliveira, Funções Especiais com Aplicaçôes, Segunda Edição, Editora Livraria da Física, São Paulo (2012).

[6] Edmundo Capelas de Oliveira, Francesco Mainardi, and Jayme Vaz Jr., Fractional models of anomalous relaxation based on the Kilbas and Saigo function, Meccanica, 49, 2049-2060 (2014).

[7] Edmundo Capelas de Oliveira, Solved Exercises in Fractional Calculus, Studies in Systems, Decision and Control, Springer, Switzerland (2019).

[8] Edmundo Capelas de Oliveira, Jayme Vaz Jr., and Stefania Jarosz, Fractional calculus via Laplace transform and its application in relaxation processes, Commun. Nonlinear Sci. Numer. Simulat., 69, 58-72 (2019).

[9] Eliana Contharteze Grigoletto, Equações Diferenciais Fracionárias e as Funções de Mittag-Leffler, Tese de Doutorado, Unicamp, Campinas, (2014).

[10] Lokenath Debnath and Dambaru Bhatta, Integral Transform and Their Applications, Chapman \& Hall/CRC, Taylor \& Francis Group, Boca Raton, (2007).

[11] M. M. Dzherbashian, On the asymptotic expansion of a function of MittagLeffler type, Akad. Nauk Armjan. SSR Doklady 19, 65-72 (1954, em Russo).

[12] M. M. Dzherbashian, On integral transform generated by the generalized Mittag-Leffler function, Izv. Akad. Nauk Armjan. SSR 13, 21-63 (1960, em Russo).

[13] Albert Edrei, Edward B. Saff and Richard Steven Varga, Zeros of Sections of Power Series, Lecture Notes in Mathematics, (A. Dold and B. Eckmann, eds), Springer-Verlag, Heidelberg, (1983).

[14] Arthur Erdélyi, Hans Heinrich Wilhelm Magnus, Fritz Oberhettinger, and Francesco Giacomo Tricomi, Higher Transcendental Functions (Bateman Manuscript Project), Volumes 1-3, McGraw-Hill Books Co., New York (1953). No Capítulo XVIII do terceiro volume, dedicado à uma miscelânea de funções, é apresentada a função de Mittag-Leffler.

[15] Rubens de Figueiredo Camargo, Jayme Vaz Jr., and Edmundo Capelas de Oliveira, On the generalized Mittag-Leffler function and its application in fractional telegraph equation, Math. Phys. Anal. Geom., 15, 1-16 (2012).

[16] Rubens de Figueiredo Camargo, Ary Orozimbo Chiacchio, and Edmundo Capelas de Oliveira, One-sided and two-sided Green?s functions, Boundary Value Problems, 2013:45 (2013).

[17] Rubens de Figueiredo Camargo e Edmundo Capelas de Oliveira, Cálculo Fracionário, Editora Livraria da Física, São Paulo, (2015). 
[18] Charles Fox, The $G$ and $H$ functions as symmetrical Fourier kernel, Trans. Amer. Math. Soc., 98, 395-429 (1961).

[19] Tord Ganelius, The zeros of the partial sums of power series, Duke Math. J. 30, 533-540 (1963).

[20] Roberto Garrappa, Numerical evaluation of two and three parameters MittagLeffler functions, SIAM J. Numer. Anal., 53, 1350-1369 (2015).

[21] Rudolf Gorenflo, Joulia Loutchko, Yuri Luchko, Computation of the MittagLeffler function $E_{\alpha, \beta}(z)$ and its derivatives, Frac. Cal. \& Appl. Anal., 5, 491518 (2002). (Corrections) 6, (2013).

[22] Rudolf Gorenflo, Anatoly Alexandrovich Kilbas, Francesco Mainardi, and Sergei V. Rogosin, Mittag-Leffler Functions, Related Topics and Applications, Springer Monographs in Mathematics, Heidelberg, (2014).

[23] Godfrey Harold Hardy, Gösta Mittag-Leffler, 1846-1927, Proc. R. Soc. London A., V-VII, 119 (1928).

[24] Hans Joachim Haubold, Arak Mathai Mathai, Ram Kishore Saxena, MittagLeffler functions and their applications, J. Appl. Math. (1110-757x) DOI: $10.1155 / 2011 / 298628$.

[25] Einar Carl Hille and Jacob David Tamarkin, On the theory of linear integral equations, Ann. Math., 31, 479-528 (1930).

[26] Pierre Humbert, Quelques résultats relatifs à la fonction de Mittag-Leffler, C. R. Acad. Sci. Paris 236, 1467-1468 (1953).

[27] Pierre Humbert, and Ravi P. Agarwal, Sur la fonction de Mittag-Leffler et quelquenes de ses généralisationes, Bull. Sci. 77, 180-185 (1953).

[28] Virginia Kiryakova, Multiindex Mittag-Leffler functions, related GelfondLeontiev operators and Laplace type integral transforms, Fract. Cal. Appl. Anal. 2, 445-462 (1999) and Multiple (multiindex) Mittag-Leffler functions and relations to generalized fractional calculus, J. Comput. Appl. Math. 118, 241-259 (2000).

[29] Édouard Louis Emmanuel Julien Le Roy, Valeurs asymptotiques de certaines séries procédant suivant les puissances entières et positives d'une variable réelle, Bull. Sci. Math. Série 2, 24, 245-268 (1900).

[30] Boris Yakovlevich Levin, Distribution of zeros of entire functions, Translations of Mathematical Monographs, Volume 5, Amer. Math. Soc,, Providence, (1964).

[31] Ernst Leonard Lindelöf, Sur le prolongement analytique, Bull. Soc. Math. de France, 29, 157-160 (1901).

[32] Axel Johannes Malmquist, Sur le calcul des integrales d'un sustème d'équations différentielles para la methodé de Cauchy-Lipschitz, Arkiv för Mat. Astr. och Fysik Bd.,1, 149-156 (1903).

[33] Arak Mathai Mathai, Ram Kishore Saxena, and Hans J. Haubold, The $H$ Function, (Theory and Applications), Springer, New York (2010).

[34] Hjalmar Mellin, Om definita integraler, Acta Soc. Scientiarum Fennicae, 20, 1-39 (1895).

[35] Magnus Gustaf (Gösta) Mittag-Leffler, Sur la représentation analytique d'une branche uniforme d'une fonction monogène, (première note) Acta Math. 23, 43-62 (1899).

[36] Magnus Gustaf (Gösta) Mittag-Leffler, Sur la représentation analytique d'une branche uniforme d'une fonction monogène, (seconde note) Acta Math. 24, 183-204 (1900).

[37] Magnus Gustaf (Gösta) Mittag-Leffler, Sur la représentation analytique d'une branche uniforme d'une fonction monogène, (troisème note) Acta Math. 24, 205-244 (1900). 
[38] Magnus Gustaf (Gösta) Mittag-Leffler, Sur la représentation analytique d'une branche uniforme d'une fonction monogène, (quatrième note) Acta Math. 26, 353-392 (1902).

[39] Magnus Gustaf (Gösta) Mittag-Leffler, Sur la représentation analytique d'une branche uniforme d'une fonction monogène, (cinquième note) Acta Math. 29, 101-181 (1905).

[40] Magnus Gustaf (Gösta) Mittag-Leffler, Sur la représentation arithmétique des fonctions analytiques d'une variable complexe, Atti del IV Congresso Internazionale dei Matematici, 1, 67-86 (1908).

[41] Magnus Gustaf (Gösta) Mittag-Leffler, Sur la représentation analytique d'une branche uniforme d'une fonction monogène, (sixième note) Acta Math. 42, 285-308 (1920).

[42] Magnus Gustaf (Gösta) Mittag-Leffler, Sur l'intégrale de Laplace-Abel, C. R. Acad. Sci. Paris 135, 937-939 (1902).

[43] Magnus Gustaf (Gösta) Mittag-Leffler, Une généralization de l'intégrale de Laplace-Abel, C. R. Acad. Sci. Paris 136, 537-539 (1903).

[44] Magnus Gustaf (Gösta) Mittag-Leffler, Sur la nouvelle fonction $E_{\alpha}(x)$, C. R. Acad. Sci. Paris 137, 554-558 (1903).

[45] Magnus Gustaf (Gösta) Mittag-Leffler, Sopra la funzione $E_{\alpha}(x)$, Rend. Acc. Lincei 13, 3-5 (1904).

[46] Magnus Gustaf (Gösta) Mittag-Leffler, Sur la représentation analytique d'une branche uniforme d'une fonction monogène, Acta Math. 29, 101-181 (1905).

[47] Daniela da Silva de Oliveira, and Edmundo Capelas de Oliveira, HilferKatugampola fractional derivative, Comput. Appl. Math., 37, DOI: 10.1007/S40314-017-0536-8 (2018).

[48] Salvatore Pincherle, Su funzioni ipergeometriche generalizzate, Atti R. Accad. Lincei Rend. Cl. Sci. Fis. Natur., 4, 694-700 e 792-799 (1888).

[49] Harry Pollard, The completely monotonic character of the Mittag-leffler function $E_{\alpha}(-x)$, Bull. Amer. Math. Soc., 54, 1115-1116 (1948).

[50] T. R. Prabhakar, A singular integral equation with a generalized Mittag-Leffler function in the kernel, Yokohama Math. 9, 7-15 (1971).

[51] B. Ramachandran, On the order and the type of entire characteristic functions, Ann. Math. Stat. 33, 1238-1255 (1962).

[52] Sergei V. Rogosin, The role of the Mittag-Leffler functions in fractional modeling, Mathematics, 3, 368-381 (2015).

[53] Paul Charles Rosenbloom, Distribution of Zeros of Polynomials, in Lectures on Functions of a Complex Variable (W. Kaplan, ed.) University of Michigan Press, Ann Arbor, 265-285 (1955).

[54] Bertram Ross, editou, Fractional Calculus and its Applications, Proceedings of the International Conference on Fractional Calculus and Applications, University of New Haven, West Haven, Conn., June 1974; Springer-Verlag, New York, (1975).

[55] Graziane Sales Teodoro, Cálculo Fracionário e as Funções de Mittag-Leffler, Disertação de Mestrado, Imecc-Unicamp, Campinas, (2014)

[56] Graziane Sales Teodoro and E. Capelas de Oliveira, Laplace transform and the Mittag-Leffler function, Int. J. Math. Educ. Sci. Technol., 45, 595-604 (2014).

[57] Graziane Sales Teodoro, Daniela da Silva de Oliviera e Edmundo Capelas de Oliveira, Sobre derivadas fracionárias, Rev. Bras. Ens. Fis., 40 (2) e2307 (2018).

[58] Graziane Sales Teodoro, José António Tenreiro Machado, and Edmundo Capelas de Oliveira, A review of definitions of fractional derivatives and other operators, J. Comput. Phys. 388, 195-208 (2019). 
[59] Giovanni Sansone and J. Gerretsen, Lectures on the Theory of Functions of a Complex Variable. Holomorphic Functions, Volume 1, Noordhoff, Groningen (1960).

[60] Rene L. Schilling, R. Song, and Zoran Vondraček, Bernstein Functions (Theory and Applications), Second Edition, De Gruyter, Berlin/Boston, (2012).

[61] José Vanterler da Costa Sousa, and Edmundo Capelas de Oliveira, On the $\psi$-Hilfer fractional derivative, Commun. Nonl. Sci. Numer. Simulat. 60, 72-91 (2018).

[62] José Vanterler da Costa Sousa, and Edmundo Capelas de Oliveira, Leibniz type rule: $\psi$-Hilfer fractional operator, Commun. Nonl. Sci. Numer. Simulat. 77, 305-311 (2019).

[63] David Vernon Widder, The Laplace Transform, Princeton University Press, Princeton, (1946).

[64] Anders Wiman, Über den Fundamentalsatz der Funkntionen $E_{\alpha}(x)$, Acta Math. 29, 191-201 (1905).

Departamento de Matemática Aplicada, instituto de Matemática, Estatística e Computação Científica, Unicamp, 13083-859, Campinas, $\mathrm{SP}$

Email address: capelas@unicamp.br 